\title{
Developing Criteria for Asian Facial Skin Health Status Based on a Non-Invasive Skin Test: The Delphi Process
}

\author{
Nan $\mathrm{Li}^{1,2}$, Mengmeng Zhao ${ }^{3}$, Xiaoxiao Yang $^{3}$, Yifan $\mathrm{He}^{3}$, Fan Yi (iD ${ }^{3}$ \\ 'Centre for Health Management and Policy Research, School of Public Health Cheeloo College of Medicine, Shandong University, Jinan, People's \\ Republic of China; ${ }^{2} \mathrm{NHC}$ Key Lab of Health Economics and Policy Research (Shandong University), Jinan, People's Republic of China; ${ }^{3}$ Key Laboratory \\ of Cosmetic, China National Light Industry, Beijing Technology and Business University, Beijing, People's Republic of China
}

Correspondence: Fan Yi, Beijing Technology and Business University, No. I I/33, Fucheng Road, Haidian District, Beijing, I00048, People's Republic of China, Email fantasyee@btbu.edu.cn

Objective: We aimed to develop a set of facial skin health criteria, combined with skin characteristics, and then determine two levels of skin condition and skin problems.

Methods: Through a literature study and group discussion, an expert consultation index system was initially formed. The Delphi method was used to conduct two rounds of expert opinion consultation. The index was revised, supplemented and reduced based on the experts' ratings and feedback, and the expert score was calculated. The authority and enthusiasm of the experts and the coordination of the expert opinions finally resulted in a new facial skin Health evaluation index system.

Results: The first-level indicators are skin problems and skin condition, the second-level indicators are detailed descriptions, and the third-level indicators are indicators that can be measured by an instrument.

Conclusion: The construction of this system can be used to quantitatively evaluate the health status of facial skin with the help of commonly used skin measuring instruments. This scientific and reasonable facial skin health evaluation index system allows people to have an objective and accurate understanding of their own facial skin health and to gradually improve their facial skin health under monitorable conditions.

Keywords: facial skin health evaluation, index system, Delphi method

\section{Introduction}

At present, most experts and doctors evaluate skin condition based on their own subjective experience. However, this method lacks accuracy and is too subjective to be an objective standard for evaluating skin.

The skin tester aims to measure a single index of the skin, and the displayed skin condition types are limited. Although the skin tester can now test multiple physiological indexes of skin, the indexes are independent of each other and cannot reflect their comprehensive effects on the skin condition. In addition, there are some measuring instruments that use different light sources to take pictures to understand skin problems. Although images can provide a basis for skin evaluation, most of them focus on the manifestation of skin problems. In summary, the subjective evaluation results are not accurate, and the objective evaluation dimensions are relatively isolated and are not conducive to a comprehensive evaluation of skin. In view of these problems, we propose two principles for the construction of a facial skin health evaluation system. Firstly, based on the knowledge of skin in previous skin Research, the comprehensive evaluation of facial skin health is carried out from the perspectives of both skin condition and skin problems. Then considering the applicability of the facial skin health evaluation system after it is established, the third-level indicators in the system are selected as objective indicators that can be measured by the current instruments.

Based on the principles of construction, this research starts from the perspective of a facial skin health evaluation in two levels: skin condition and skin problems. Through a layer-by-layer decomposition of the goal of a facial skin health 
evaluation, the goals in the eight dimensions of barrier, skin colour, skin quality, pores, acne, pigmentation, wrinkles, and sensitivity are determined based on the characteristics of these dimensions and the relationships between them. Thirteen indicators are designed to characterize skin condition, and 19 indicators are designed to characterize skin problems, thus forming a preliminary facial skin health evaluation indicator system. In this paper, the Delphi method is used to screen the indicators to obtain key indicators that can show the degree of facial skin health and finally form a facial skin health evaluation index system.

\section{Materials and Methods}

\section{Preliminary Drafting of the Indicator System} Establishing a Coordination Group

The coordination group consisted of 3 members, 1 master supervisor and 2 master students. The main tasks of the coordination group were to consult the literature, form a preliminary facial skin health evaluation index system, select experts, design and issue consultation questionnaires, perform statistics, sort and analyze the results of the questionnaire, and finally determine the evaluation index system. The whole process does not involve human experiments, so there is no ethics statement. However, the relevant ethics numbers for the human facial skin index collection as below: The study was approved by the Ethics Committee of Xiyuan Hospital of China Academy of Chinese Medical Sciences (2019XL013-2) and was registered in the Chinese Clinical Trial Registry (registration number: ChiCTR1900025405). All healthy volunteers consented to participate in writing before enrollment.

\section{Principles of Index Design}

The first step in building a facial skin health evaluation system is to design evaluation indicators. The quality of the evaluation indicators is directly related to the feasibility of the facial skin health evaluation system. To ensure the scientificity, rationality and feasibility of the facial skin health evaluation system, on the basis of consulting expert opinions and combining the characteristics of the skin, the facial skin health evaluation index system was designed according to the following principles:

(1) The indicators are comprehensive. ${ }^{1}$ The design of the indicator system should cover the basic content of the evaluation as much as possible. If something is omitted, the evaluation will be biased. This requires the selected indicators to be representative of different aspects. The selected indicators should be focused on all aspects of the things being evaluated. Although there may not be many final evaluation indicators, in the initial selection, the candidate indicators must be increasingly comprehensive to ensure room for selection. In view of this principle, the CK indicators that characterize the skin condition were selected, which are the moisture content CM at the barrier level, the water loss TEWL, oil content SM, $\mathrm{pH}$ value, elasticity R2, elasticity R5, and elasticity R7 at the skin quality level, and the gloss at the skin tone level. The degree GLOSS_DSC, melanin content MEXA, heme content ERYTH, chromaticity values b (ie, SP_LAB3) and a (ie, SP_LAB2), and individual typology angle (ITA) value (SP_ITA). The indicators transformed by VISIA, which characterizes skin problems, were pores, acne, stains, wrinkles, red blood streaks, dark circles, and eye bags.

(2) The indicators are independent. ${ }^{1}$ The establishment of the evaluation index system should choose relatively independent evaluation indexes; that is, each evaluation index in the index system must meet the requirement for relative independence from the others as much as possible while conforming to the principle of integrity. It is required that the selected indicators be relatively independent, and there should be no inclusion and little overlap and similarity to avoid duplication of information as much as possible. However, this kind of independence is also relative. It is necessary to meet the requirements of independence as well as possible, but it is impossible to achieve complete independence. The degree of independence must match the selected evaluation method. The final selected indicators should satisfy the principle of independence as well as possible. In view of this principle, elasticity R5 and elasticity R7 were deleted, and only the total elasticity $\mathrm{R} 2$ was retained.

(3) The indicators are feasible. ${ }^{1}$ The indicators should be feasible, in line with objective and practical levels, have stable data sources, be easy to operate, and have a clear indicator meaning, standardized data, consistent calibre, and simple and easy data collection. Based on this principle, all third-level indicators selected for the construction of the facial skin health evaluation system can be measured by instruments, and the meaning of each indicator is clear. 


\section{Preliminary Formation of the Facial Skin Health Evaluation Index System}

Based on existing experience, the coordination group formed a preliminary facial skin health evaluation system based on the principles of facial skin health evaluation system construction and indicator design principles and by consulting relevant literature and data on facial skin health evaluation. The system included 2 first-level indicators, 8 second-level indicators and 30 third-level indicators. The various indicators that characterize the skin condition were tested by the CK instrument. Each volunteer's skin hydration in the stratum corneum (CM), skin melanin content (MEXA), erythema indexes(ERYTH), and skin gloss index(GLOSS_DSC) were repeated 5 times on the left and right sides of the face, for a total of 10 measurements. The average value of the 10 measurements was then obtained. The $\mathrm{pH}$ of the skin surface( $\mathrm{pH})$, transepidermal water loss (TEWL), sebum content(SM), skin elasticity(R2), yellowness b value, redness a value, individual typology angle ITA were measured 3 times for both the left and right sides of the face, and then the average value of the 6 measurements was obtained.In our previously research. ${ }^{2-6}$, we focused on utilize these facial skin test to unveil the regulation of facial aging progress and other The methodology by use CK instruments and statistical analysis. The indicators that characterize skin problems were extracted by algorithms after taking pictures with the multilight source skin imager VISIA. The specific indicators and their descriptions are shown in Table 1.

\section{Expert Selection}

According to the theoretical requirements of the Delphi method, the number of experts can be determined according to the size of the research project and the width of the involved area, generally approximately 8-20 persons. ${ }^{7,12}$ To follow the principle of combining representativeness and authority, 10 experts were selected for this study.

\section{Design Letter Inquiry Sheet}

The questionnaire consisted of 4 parts: (1) Questionnaire description: this introduces the background, purpose, significance, questionnaire filling method and feedback time of this research; (2) Questionnaire subject: experts use a Likert scale $^{8,13}$ to score the importance of the indicators at 5 levels, from "very unimportant" to "very important", each with a score of 1 to 5, and an additional index column and an index modification comment column are set; (3) General situation survey form for the experts: the survey concerns gender, age, working years, professional title, education background, work unit, work field, etc.; (4) Experts' familiarity and judgement basis self-evaluation table: ${ }^{9,14,15}$ familiarity is divided into very unfamiliar (0.2), unfamiliar (0.4), generally familiar (0.6), familiar (0.8), and very familiar (1). There are 4 judgement bases, each of which can be divided into three levels (large, medium and small): practical experience $(0.5,0.4,0.3)$, theoretical analysis $(0.3,0.2,0.1)$, domestic and foreign reference materials $(0.1,0.1$, $0.1)$, and intuition $(0.1,0.1,0.1)$ (Table 2).

\section{The Specific Implementation of the Delphi Method}

The researcher contacted the experts in advance to explain the research background and purpose and conducted two rounds of Delphi letter inquiries to the 10 experts. In this method, the questionnaire is issued to each expert by e-mail or paper submission, and the expert is requested to reply within 2 weeks if possible. After the first round of expert letter questionnaires were received, according to the screening criteria, the experts' feedback opinions were synthesized and sorted, and the content of the indicators was modified and adjusted to form the second round of questionnaires. The experts were then consulted again through statistical analyses and expert opinions. When there was a high degree of consistency and good coordination, there was no need for the next round of letter inquiries, and the results of the last consultation were used as the final evaluation index. According to the principle of the Delphi method, this research conducted a total of 2 rounds of investigation. The Delphi process is shown in Figure 1.

\section{Statistical Indicators}

For the basic expert information, such as academic qualifications, title, position, and working years, a descriptive analysis was performed to determine characteristics such as frequency, composition ratio, mean, and standard deviation.

Experts' positive coefficient: This is the recovery rate of the expert consultation table (recovery rate $=$ the number of experts involved/the number of all experts), and its size indicates how much the experts care about the project. 
Table I Index Description

\begin{tabular}{|c|c|c|c|c|}
\hline $\begin{array}{l}\text { First-Level } \\
\text { Indexes }\end{array}$ & $\begin{array}{l}\text { Second-Level } \\
\text { Indexes }\end{array}$ & Test Instrument & Third-Level Indexes & Index Description \\
\hline $\begin{array}{l}\text { Facial skin } \\
\text { condition }\end{array}$ & $\begin{array}{l}\text { Skin Barrier } \\
\text { Skin quality } \\
\text { Colour } \\
\text { Pores } \\
\text { Acne } \\
\text { Pigmentation } \\
\text { Sensitive }\end{array}$ & $\begin{array}{l}\text { Skin-pH-Meter PH905 } \\
\text { Corneometer CM } 825 \\
\text { Tewamater TM300 } \\
\text { Sebumeter SM8I5 } \\
\text { Cutometer dual MPA580 } \\
\text { GL200 } \\
\text { Mexameter MXI8 } \\
\text { Colorimeter CL400 } \\
\text { VISIA picture algorithm } \\
\text { extraction }\end{array}$ & $\begin{array}{l}\text { PH } \\
\text { CM } \\
\text { TEWL } \\
\text { SM } \\
\text { R2 } \\
\text { GLOSS_DSC } \\
\text { MEXA } \\
\text { ERYTH } \\
\text { b value (ie, SP_LAB3) } \\
\text { a value (ie, SP_LAB2) } \\
\text { ITA value (SP_ITA) } \\
\text { Number of pores } \\
\text { Number of blackheads } \\
\text { Number of acne } \\
\text { Number of nodules } \\
\text { Number of pustules } \\
\text { Number of acne marks } \\
\text { Number of chloasma } \\
\text { Number of freckles } \\
\text { Number of sunburn } \\
\text { Number of age spots } \\
\text { Number of UV spots } \\
\text { Number of brown spots } \\
\text { Number of horizontal forehead } \\
\text { wrinkles } \\
\text { Number of glabellar wrinkles } \\
\text { Number of periorbital wrinkles } \\
\text { Number of crow's feet } \\
\text { Number of nasolabial folds } \\
\text { Number of perioral wrinkles } \\
\text { Red blood streak (percentage) }\end{array}$ & $\begin{array}{l}\text { The } \mathrm{pH} \text { of the skin surface, generally considered to be in the range of } 4.5-6.5 \\
\text { Skin hydration in the stratum corneum. The greater the CM value, the greater the moisture } \\
\text { content of the skin } \\
\text { Transepidermal water loss.The larger the TEWL, the worse the skin barrier }{ }^{7} \\
\text { Sebum content.The greater the SM value, the more sebum content } \\
\text { Skin elasticity. R2 value in the range of } 0-1 \text {; the larger the value, the better the elasticity } \\
\text { Skin gloss index. The greater the index value, the higher the gloss } \\
\text { Skin melanin content. The larger the index value, the darker the skin tone } \\
\text { Erythema indexes. The larger the index value, the redder the skin colour } \\
\text { Yellownessb value. The greater the b value, the higher the yellowness of the skin } \\
\text { Redness a value. The greater the value of a, the higher the redness of the skin } \\
\text { Individual typology angle ITA. The greater the ITA value, the lighter the skin tone }{ }^{10,11} \\
\text { Represents different skin problems; the larger the index value, the more serious the } \\
\text { corresponding skin problem }\end{array}$ \\
\hline
\end{tabular}


Table 2 Experts' Judgement Basis and Familiarity Quantification Table for Indicators

\begin{tabular}{|l|c|c|c|c|c|}
\hline \multirow{2}{*}{ Judgement Bases (Ca) } & \multicolumn{3}{|c|}{ Quantized Value } & \multirow{2}{*}{ Familiarity (Cs) } & Quantized Value \\
\cline { 2 - 4 } & Big & Middle & Small & & \\
\hline Experience & 0.5 & 0.4 & 0.3 & Very familiar & I \\
Theoretical analysis & 0.3 & 0.2 & 0.1 & Familiar & 0.8 \\
References at home and abroad & 0.1 & 0.1 & 0.1 & Generally familiar & 0.6 \\
Intuition & 0.1 & 0.1 & 0.1 & Unfamiliar & 0.4 \\
& & & & Very unfamiliar & 0.2 \\
\hline
\end{tabular}

Degree of authority of the expert opinions: The degree of authority of an expert is generally determined by two factors: one is the basis for the expert to make a judgement on the plan, and the other is the degree of familiarity of the expert with the problem. The authority of experts is mainly based on self-evaluation. The expert authority coefficient $(\mathrm{Cr})$ is expressed as the arithmetic mean of the familiarity coefficient $(\mathrm{Cs})$ and judgement basis coefficient $(\mathrm{Ca})$ : authority $=$ (familiarity coefficient + judgement coefficient) $/ 2, \mathrm{ie}, \mathrm{Cr}=(\mathrm{Cs}+\mathrm{Ca}) / 2 . \mathrm{Cr} \geq 0.7$ indicates that it is authoritative. ${ }^{10,11,14,16}$

Concentration of the expert opinions: The concentration of expert opinions is expressed by the mean. The mean value is $0-9$ or $0-100$ points. The larger the value is, the higher the importance of the corresponding index. The full score ratio is between 0 and 1, and it can be used as a supplementary indicator to the average. The larger the full score ratio, the greater the percentage of experts who give full marks to the indicator, and the more important the indicator is. Coordination degree of the expert opinions: This is expressed by the coefficient of variation and the coordination coefficient.

The coefficient of variation indicates the degree of coordination on the index by experts. The smaller the coefficient of variation $\left(\mathrm{CV}=\right.$ standard deviation/mean), the higher the degree of coordination; it is usually $<0.25 .{ }^{12,17}$ The coordination coefficient indicates the degree of coordination of all experts for all indicators. The larger the coordination degree of the expert opinions, the higher the coordination coefficient (W) obtained by the nonparametric Kendall $W$-test of multiple samples. The value of $\mathrm{W}$ is between 0 and 1 . The larger $\mathrm{W}$ is, the higher the degree of coordination. By calculating the coefficient of variation and the coordination coefficient, it can be determined whether there are large differences in the evaluation of each indicator by the experts, or whether there are highly coordinated experts and experts with heretical opinions. The coefficient of variation, the coordination coefficient and the significance test of the coordination coefficient can all be calculated by SPSS 25.0 statistical software.

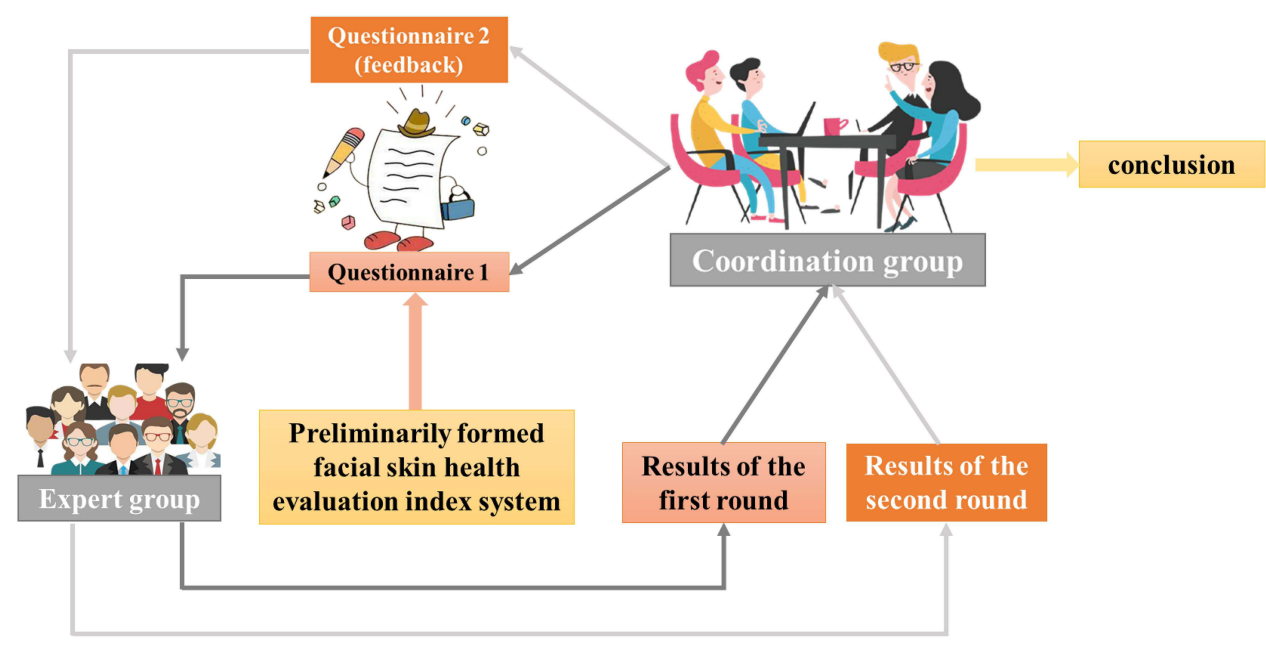

Figure I Delphi expert questionnaire letter inquiry process. 


\section{Statistical Methods}

Using SPSS 25.0 statistical software, the statistical indicators included the basic situation of the experts, the positive coefficient of the experts, the concentration of the expert opinions, the coordination degree of the expert opinions and the authority of the experts; for the calculation methods of each indicator, refer to the relevant literature. ${ }^{9,13,18,19}$

\section{Screening Criteria for Indicators}

The boundary value method was used to screen the evaluation indicators. ${ }^{14,15}$ According to the importance scores of each evaluation index by the experts, the average value, full score ratio and coefficient of variation of each index were calculated, and the indexes were screened according to their boundary values.

The cut-off value refers to the calculation of the mean value, the full score ratio and the coefficient of variation of all the indicators, and on this basis, the arithmetic mean and standard deviation of the mean value of the indicators, the full score ratio and the coefficient of variation were calculated. (1) Among them, the boundary value of the index mean and full score frequency $=$ arithmetic mean - standard deviation, and the boundary value of the coefficient of variation $=$ arithmetic mean + standard deviation. When any of the following conditions occurred in the indicator, it was recommended to delete it: one is that for the mean and full score, the frequency is lower than the corresponding threshold and the coefficient of variation is higher than the threshold; the second is that the mean and full score frequencies are lower than the corresponding threshold and the coefficient of variation is lower than the threshold. (2) Based on the indicators that were suggested to be eliminated based on the boundary value method, combined with the actual situation of the facial skin health evaluation, the project team was consulted as to whether to delete each indicator. At the same time, the index selection process fully considered the modification opinions put forward by the experts.

\section{Results}

\section{Basic Situation of the Experts}

A total of 10 experts were included, with an average age of 39.7 years, and $90 \%$ of them had a postgraduate degree. The work areas involved included skin research, cosmetic safety and efficacy evaluation, cosmetic research and application, and consumer and market research. The average working time was 14.5 years. For details of this information, see Table 3 .

\section{Enthusiasm of the Experts}

The enthusiasm of the experts is expressed by the effective response rate to the questionnaire. The effective recovery rate of the first round of questionnaires was 100\% (10 copies were issued, and 10 copies were returned); the effective recovery rate of the second round of questionnaires was $100 \%$ (10 copies were issued and 10 copies were recovered), indicating that the experts were concerned about and invested in this research and were highly motivated.

\section{Authority of the Experts}

In the two rounds of questionnaires, the authority coefficients of the 10 consulting experts were all greater than 0.7 , and the mean values were 0.845 and $0.85(>0.7)$, respectively. The 10 consulting experts that participated in this study had high authority (Table 4).

\section{Expert Concentration}

The degree of concentration of the experts is represented by the mean of the importance of the indicators and the coefficient of variation (the table below). The coefficient of variation in the first round was $C V=0.1652$, and the coefficient of variation in the second round was $C V=0.1640$, both of which are less than 0.25 , indicating the concentration of expert opinions (Table 5).

\section{Degree of Coordination of the Expert Opinions}

In the first round of this research, the expert coordination coefficient of the first round of consultation was 0.238. After statistical testing, $\mathrm{P}<0.001$, and the experts' coordination coefficient in the second round of consultation rose to 0.324 , 
Table 3 Basic Situation of the Experts $(n=10)$

\begin{tabular}{|l|c|c|c|}
\hline Project & Classification & $\begin{array}{c}\text { Number of } \\
\text { People }\end{array}$ & $\begin{array}{c}\text { Composition } \\
\text { Ratio (\%) }\end{array}$ \\
\hline Age & $20 \sim<30$ years old & 3 & 30 \\
& $30 \sim<40$ years old & 3 & 30 \\
Gender & $40 \sim<50$ years old & 1 & 10 \\
& $50 \sim<60$ years old & 3 & 30 \\
Education & Male & 3 & 30 \\
& Female & 7 & 70 \\
Working years & Undergraduate & 1 & 10 \\
& Master & 5 & 50 \\
& PhD & 4 & 40 \\
Field of work & $1 \sim<10$ years old & 4 & 30 \\
& I0 <20 years old & 1 & 10 \\
& $20 \sim<30$ years old & 2 & 20 \\
& $30 \sim<40$ years old & 3 & 30 \\
& Skin research & 2 & 20 \\
& Research and application of cosmetics (Traditional & & \\
& Chinese Medicine) & 2 & 30 \\
\hline
\end{tabular}

Table 4 Expert Authority Status Across the Two Rounds $(n=10)$

\begin{tabular}{|l|c|c|}
\hline \multirow{2}{*}{ Number } & Round One & Round Two \\
\cline { 2 - 3 } & Cr=(Cs+Ca)/2 & Cr=(Cs+Ca)/2 \\
\hline 1 & 0.95 & 1 \\
2 & 0.8 & 0.8 \\
3 & 0.95 & 0.95 \\
4 & 0.9 & 0.95 \\
5 & 0.8 & 0.8 \\
6 & 0.8 & 0.8 \\
7 & 0.85 & 0.85 \\
8 & 0.8 & 0.75 \\
9 & 0.8 & 0.8 \\
10 & 0.8 & 0.8 \\
Average & 0.845 & 0.85 \\
\hline
\end{tabular}

Table 5 Expert Importance Assignment Situation Across the Two Rounds $(n=10)$

\begin{tabular}{|l|c|c|c|}
\hline Round & Average & Standard Deviation & Coefficient of Variation \\
\hline Round one & 4.32 & 0.699 & 0.1652 \\
Round two & 4.13 & 0.660 & 0.1640 \\
\hline
\end{tabular}

$\mathrm{P}<0.001$, indicating that the evaluation results of the experts on the indicators tended to be consistent and the evaluation results were desirable. See the Table 6 below for details.

\section{Index Screening Results}

In the first round of correspondence, the indicators recommended to be deleted according to the boundary value method were the second-level index pores, the third-level index blackheads, and the number of age spots. However, because large 
Table 6 Expert Opinion Coordination Across the Two Rounds $(n=10)$

\begin{tabular}{|l|c|c|c|}
\hline Round & Coordination Coefficient (Kendall's W) & Chi-Square Value $(\boldsymbol{\alpha} \mathbf{2})$ & $\boldsymbol{P}$ \\
\hline Round one & 0.238 & 96.653 & 0.000 \\
Round two & 0.324 & 126.263 & 0.000 \\
\hline
\end{tabular}

pores, blackheads and age spots are common skin problems, it was decided to keep these three indicators after a group discussion. After the first round of expert consultation, expert opinions were adopted, and the third-level indicator $\mathrm{L}$ value was increased. Because ITA was calculated by LAB and considering the independence principle of indicator design, the indicator ITA was deleted. The results of the second round of expert correspondence indicated that no indicators need to be modified or deleted.vRefer to Table 7 for details of the boundary value table of the two rounds of expert consultation and the selection indicators.

In summary, the final facial skin health evaluation index system is shown in the Table 8, including 2 first-level indicators, 8 second-level indicators, and 30 third-level indicators. In addition to the facial skin health evaluation index system, age should be considered in the comprehensive evaluation of facial skin health.

\section{Discussion}

\section{Content Analysis of the Facial Skin Health Evaluation Index System}

(1) Comprehensiveness of the evaluation indicators

The construction of the facial skin health evaluation system follows the principle of comprehensive index design. To ensure the objectivity of the evaluation, the index system covers the basic content of the evaluation as much as possible, and the selected indexes are representative in different respects. The selected indicators focus on all aspects of facial skin health, and finally, the CK indicators were selected to characterize the skin condition, which are skin hydration in the stratum corneum (CM), transepidermal water loss (TEWL), sebum content (SM), the $\mathrm{pH}$ of the skin surface at the barrier level, Skin elasticity (R2) at the skin quality level, skin gloss index(GLOSS_DSC), Skin melanin content (MEXA), erythema indexes (ERYTH), brightness L(ie, SP_LAB1), redness a(ie, SP_LAB2), yellowness b(ie, SP_LAB3), The indicators transformed by VISIA, which characterize common skin problems, are pores, acne, stains, wrinkles, and sensitivity.

(2) The objectivity of the evaluation system

When determining the index system, this article consulted a large number of studies based on the existing understanding of skin from previous skin state research, consulted the opinions and suggestions of 10 experts, and comprehensively considered the selected evaluation index to be more objective.

\section{Scientificity and Reliability of the Facial Skin Health Evaluation Index System}

In the initial stage of this research, based on the comprehensiveness, feasibility and independence principles among the index design principles and taking into account the applicability of the facial skin health evaluation index system after the completion of the evaluation index system, the coordination team performed a preliminary study on the basis of a large amount of literature and

Table 7 Boundary Value Table for the Two Rounds of Expert Consultation and the Selection Indexes

\begin{tabular}{|l|c|c|c|c|c|c|}
\hline Rounds & \multicolumn{3}{|c|}{ Round One } & \multicolumn{3}{c|}{ Round Two } \\
\hline & $\begin{array}{c}\text { Average } \\
(\bar{x})\end{array}$ & $\begin{array}{c}\text { Full Score } \\
\text { Ratio (K) }\end{array}$ & $\begin{array}{c}\text { Coefficient of } \\
\text { Variation (CV) }\end{array}$ & $\begin{array}{c}\text { Average } \\
(\bar{x})\end{array}$ & $\begin{array}{c}\text { Full Score } \\
\text { Ratio (K) }\end{array}$ & $\begin{array}{c}\text { Coefficient of } \\
\text { Variation (CV) }\end{array}$ \\
\hline First-level index boundary value & 4.779 & 0.779 & 0.093 & 4.638 & 0.638 & 0.124 \\
Second-level index boundary value & 4.193 & 0.344 & 0.192 & 4.066 & 0.193 & 0.173 \\
Third-level index boundary value & 4.066 & 0.265 & 0.206 & 3.762 & 0.073 & 0.250 \\
\hline
\end{tabular}


Table 8 Facial Skin Health Evaluation Index System

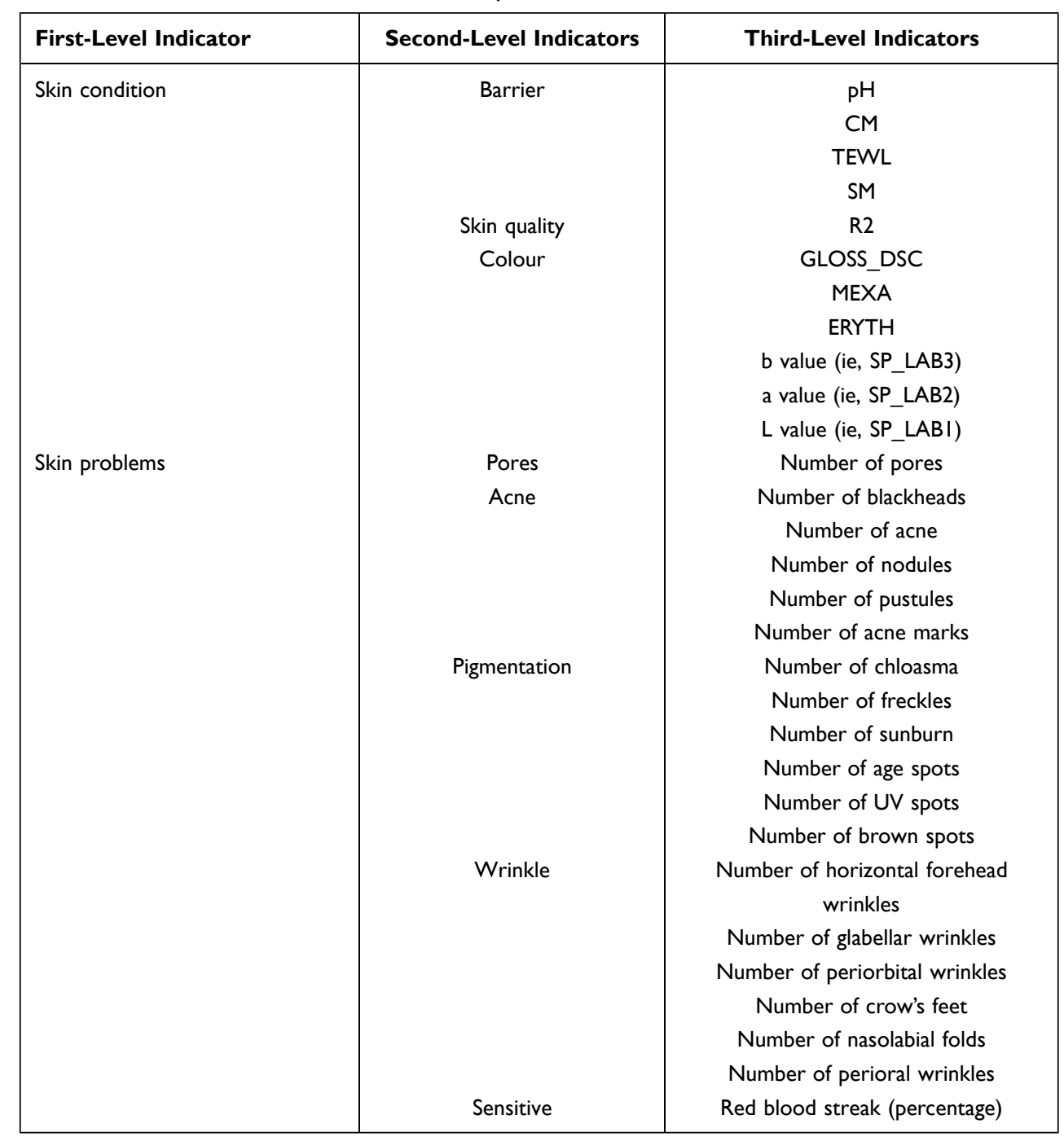

existing experience. A facial skin health evaluation index system was formed, and then the Delphi method was used for screening to determine the final facial skin health evaluation index system.

The Delphi method, also known as the expert consultation method, is a method of judging opinions and value through several rounds of expert consultation. It uses the knowledge, wisdom, experience, and information of a number of experts to evaluate the evaluation indicators that have been proposed. Carrying out analyses and judgements, weighing and assigning corresponding importance values, breaking through the limitations of traditional quantitative analyses, and obtaining more representative and reliable results compared with other empirical forecasting methods give the Delphi method more practical value.

This research adopted the Delphi expert consultation method to conduct 2 rounds of correspondence inquiries with 10 experts, all of whom have a bachelor's degree or above and have worked for more than 5 years. They have in-depth and comprehensive work content and specific requirements related to skin and cosmetics. The effective recovery rate of the two rounds of questionnaires was $100 \%$, and the experts' authority coefficients were 0.845 and 0.85 , both greater than 0.7 , indicating that the experts in this study had good authority. The coordination coefficients of the expert opinions were 0.238 and 0.324 , respectively, and the significance test results were all $<0.001$, which indicates that the expert opinions tended to be unanimous.

After 2 rounds of correspondence, a facial skin health evaluation index system consisting of 2 first-level indicators, 8 second-level indicators, and 30 third-level indicators was finally constructed. At the same time, when evaluating facial skin health, we should also consider eliminating the influence of age. 


\section{Significance of the Construction of the Facial Skin Health Evaluation Index System}

This research starts from the perspective of a comprehensive evaluation of facial skin health, is based on a skin state database, uses relevant methods of mathematical statistical analysis, and is based on reading a large number of studies from around the world and borrowing from the existing research results from the two major perspectives of skin condition and skin problems. Starting with the dimensions of the system, the Delphi expert consultation method was used to construct a comprehensive and effective evaluation index system for facial skin health, thereby laying a foundation for an objective and quantitative comprehensive evaluation of facial skin health, supplementing the deficiencies of facial skin health evaluation methods and systems, and satisfying general guiding principles. The public's demand for healthy skin care provides the cosmetics industry and consumers with a new facial skin health evaluation index system. The research direction of facial skin health is also appropriate for exploration, expansion and in-depth study.

\section{Conclusion}

In summary, the facial skin health evaluation index system constructed by the Delphi method in this study is used to comprehensively evaluate facial skin health from two perspectives: skin condition and skin problems, and it includes primary, secondary and tertiary indicators. The first-level indicators are the two major levels of skin condition and skin problems, and the second-level indicators are the subdivision of the two major levels of the first-level indicators and the description of the third-level indicators, including barrier, skin colour, skin quality, pores, acne, and pigmentation. Among the eight dimensions of wrinkles and sensitivity, the third-level indicators are specific indicators that can be measured by the instrument included in each dimension of the second-level indicators. The facial skin health evaluation system constructed from this can finally guide the general public to quantitatively evaluate their own facial skin health through instruments and equipment and, at the same time, can provide a new facial skin health evaluation system for the cosmetics industry and consumers.

\section{Disclosure}

The authors report no conflicts of interest in this work.

\section{References}

1. Yunzhong L, Yuan H. Statistical Comprehensive Evaluation Method and Application. Tsinghua University Press; 2020.

2. Wu Y, Yi F, Akimoto M, Tanaka T, Meng H, Dong Y. Objective measurement and comparison of human facial skin color in East Asian females. Skin Res Technol. 2020;26(4):584-590. doi:10.1111/srt.12838

3. Li N, Yang XX, Yang RY, Yi F. Study of the characteristics of facial skin tone status in 1092 young Chinese females according to the ITA. $J$ Cosmet Dermatol. 2021. doi:10.1111/jocd.14356

4. Wei SY, Zhang HY, Yin YT, et al. Factor analysis approach unveils the influencing factors of dandruff in the normal teenage population. Dermatol Ther. 2020;33(4):e13690. doi:10.1111/dth.13690

5. Meng H, Lin W, Dong Y, et al. Statistical analysis of age-related skin parameters. Technol Health Care. 2021;29(S1):65-76. doi:10.3233/THC-218007

6. Meng H, Yin Y, Wu W, et al. Raman spectroscopic analysis of skin penetration and moisturizing effects of Bionics vernix caseosa cream compared with Vaseline. Technol Health Care. 2021;29(S1):327-334. doi:10.3233/THC-218030

7. Xin Z. Application of TEWL in evaluation of skin barrier function. Guangdong Chem Industry. 2014;41(14):133-134.

8. Yuqing Q, Quanzhong L. Elastic fiber and skin aging. Int J Dermatovenereol. 2014;40(2):97-100.

9. Shiyi W, Mingliang X, Hong M, et al. Analysis of factors influencing skin color based on ITA value. Asian J Beauty Cosmetol. 2019;2019(2):33-40.

10. Chardon A, Cretois I, Hourseau C. Skin colour typology and suntanning pathways. Int J Cosmet Sci. 2010;13(4):191-208. doi:10.1111/j.14672494.1991.tb00561.x

11. Wei L, Xuemin W, Wei L, et al. Determination and classification of skin color of 407 women in four cities. Chinese Journal of Dermatology. 2005;38(12):772-773.

12. Guoxiang X. Statistical Forecasting and Decision Making. Shanghai University of Finance and Economics Press; 1998.

13. Laibin Q. Statistical analysis and fuzzy comprehensive evaluation of Likert scale. Shandong Sci. 2006;19(2):18-23.

14. Min X, Sha M, Wei L, et al. Research on the weights of the evaluation index system of health supervision efficiency based on Delphi methodTaking Shanghai Health Supervision Institution as an example. Chin J Health Inspect. 2014;21(003):212-218.

15. Yanran L, Jie N, Ming X, et al. Research on evaluation index system of county comprehensive medical reform based on Delphi method. Hygiene Soft Sci. 2019;33(01):29-34.

16. Sapin C, Simeoni MC, Khammar ME, et al. Reliability and validity of the VSP-A, a health-related quality of life instrument for ill and healthy adolescents. J Adolesc Health. 2002;36(4):327-336. doi:10.1016/j.jadohealth.2004.01.016

17. Yujie G, Didi Y, Xuemin H, et al. Using Delphi method to construct evaluation index system of public health personnel's core competence. China Public Health Manag. 2016;2016(6):771-773.

18. Guang Z. Modern Epidemiological Methods and Applications. Joint Press of Beijing Medical University and China Peking Union Medical University; 1994.

19. Cong C, Yizhi L, Rude W. Kendall coordination coefficient W test and its SPSS realization. J Taishan Med Coll. 2010;31(007):487-490. 


\section{Publish your work in this journal}

Clinical, Cosmetic and Investigational Dermatology is an international, peer-reviewed, open access, online journal that focuses on the latest clinical and experimental research in all aspects of skin disease and cosmetic interventions. This journal is indexed on CAS. The manuscript management system is completely online and includes a very quick and fair peer-review system, which is all easy to use. Visit http://www. dovepress.com/testimonials.php to read real quotes from published authors.

Submit your manuscript here: https://www.dovepress.com/clinical-cosmetic-and-investigational-dermatology-journal 